\title{
EL PROJECT FINANCE
}

\section{PROJECT FINANCE}

\author{
Marco Antonio Trigoso SuÁrez ${ }^{*}$
}

\section{Resumen}

Tanto la actividad privada como la desarrollada por la administración pública juegan un rol trascendente para la implementación de proyectos en los que se utiliza project finance como sistema de financiación. Los vínculos se darán en todo momento, desde que se constituye la SPV pues necesitara para su funcionamiento inscribirse en el registro de personas jurídicas; el proyecto también requerirá diversas autorizaciones, por ejemplo las exigidas en el régimen de la competencia; así como licencias emitidas por las municipalidades, gobiernos regionales o centrales; la administración

* Magíster en Derecho Empresarial por la Universidad Autónoma de Madrid, España. Abogado y Bachiller por la Facultad de Derecho y Ciencia Política de la Universidad Nacional Mayor de San Marcos. Profesor ordinario y Secretario Académico y Administrativo en la Facultad de Derecho y Ciencia Política de la Universidad Ricardo Palma. La cátedra universitaria la imparto en los cursos de Títulos Valores, Mercado de Valores y Derecho Corporativo, en la universidad Ricardo Palma, UIGV (2013-14) y San Ignacio de Loyola (2014-16). Asesor legal en minería, empresarial y gestión pública. también está presente en lo referente al régimen tributario y el correspondiente pago de impuestos; incluso, en algunos casos el Estado es quien determina el control de cambio ; asimismo, los gobiernos tienen una vital influencia respecto al riesgo político o riesgo país, lo que implica un ámbito de evaluación que realizan los promotores respecto a la estabilidad del proyecto en el tiempo.

\section{Palabras clave}

Project finance - Comercio - Garantías Seguridad - Financiamiento - Infraestructura

\section{Abstract}

Both private and public activities play a transcendent role for the implementation of projects in which project finance is used as a financing system. The links will be given at all times, since the VPS is constituted as it will need to be registered in the register of legal entities for its operation; the project will also require various authorizations, for example those required under the competition regime; as well as licenses issued by municipalities, regional or central governments; the administration is 


\section{Marco Antonio Trigoso SuÁrez}

also present regarding the tax regime and the corresponding tax payment; even, in some cases, the State determines change control; In addition, governments have a vital influence on political risk or country risk, which implies an area of evaluation that the promoters make regarding the stability of the project over time.

\section{Keywords}

Project finance - Trade - Guarantees - Security - Financing - Infrastructure

\section{Nociones preliminares}

La presente investigación tiene dos propósitos, primero, ofrecer a los promotores de proyectos de infraestructura de gran envergadura, entidades financieras, proveedores, constructores, abogados y estudiantes de Derecho, un alcance general acerca de la modalidad de financiamiento de estos proyectos, "project finance"; y, segundo, contribuir con el fortalecimiento del circuito económico-jurídico-social, pues, la ejecución de estos proyectos de infraestructura no sólo genera un beneficio para los interesados directos, sino y, sin lugar a dudas, también cumple un significativo rol social, toda vez que, es el propio Estado quien expandirá su base de ingresos a través de la recaudación de impuestos y, la colectividad en su conjunto al generarse un dinamismo laboral y, como consumidores finales, el acceso competitivo los distintos bienes y servicios que ofrecerán estos proyectos.

\section{¿Qué es el "Project finance"?}

Es un mecanismo de financiación utilizado para viabilizar proyectos de grandes dimensiones. Es considerado un método de financiación alternativo que permite a los promotores de proyectos transferir parte de los riesgos inherentes de un proyecto a los bancos o a los mercados de deuda. ${ }^{1}$ Siendo, su principal características que, la devolución del monto financiado (el principal) e intereses, dependerá de los ingresos futuros que el propio proyecto genere al entrar en la etapa de funcionamiento, es decir una financiación que tiene como principal garantía el propio proyecto. Este sistema se estructura a través de cuatro rasgos principales, primero, esta financiación se basa en la identificación y asignación de los riesgos del proyecto; segundo, la financiación debido a su gran volumen será otorgada por una o varias entidades bancarias quienes asumen como principal garantía al propio proyecto; tercero, la devolución del principal y los intereses de la financiación dependerán fundamentalmente de los flujos de caja futuros que el proyecto genere, y cuarto, proteger el patrimonio de los promotores aislándolo en una sociedad independiente tanto legal como económicamente.

\section{Antecedentes}

Los primeros casos en los que se observa la financiación de proyectos se remonta a Europa a los comerciantes griegos que financiaban las expediciones de determinados navíos ${ }^{2}$. En el Reino Unido surge con el fin de incorporar el financiamiento privado al sector público. La primera concesión que conócenos tuvo lugar en 1554, en Francia, se le otorgo a Adam de Craponne la construcción de un canal entre los ríos Durnace y Rhone, esta fue la precursora de los contratos BOT (Build Own Transfer). La aparición del ferrocarril, a principios del siglo XIX, dio lugar a otra forma de financiamiento de proyectos: la emisión de obligaciones o acciones.

1 ICEX, Instituto Español de Comercio Exterior, "Project Finance, Financiamiento de Proyectos Internacionales”, Madrid, 2002, pág. 15

2 Vaño Vaño, Maria José, "El Contrato de Project Finance”, Tirant lo Blanch, Valencia 2002, p. 35 
No obstante, se considera que la modalidad de financiación de proyectos que se conocen en la actualidad se originó en los años treinta del siglo XX, en los campos de petróleo de Texas, para financiar gastos aparejados a la inversión. El perforador financiaba los costes de la perforación a cambio de un porcentaje de los ingresos procedentes de la venta del petróleo. En aquella época - y en la zona oeste de Texas - era difícil no extraer petróleo cada vez que se perforaba, por lo que un banco de Dallas concedió un préstamo sin recurso para la explotación de un campo de gas y petróleo, que se cancelo mediante los flujos de caja procedentes de los pozos. En los años sesenta, las operaciones relacionadas con los recursos naturales - especialmente minería, petróleo, gas - se ampliaron, impulsadas principalmente por los bancos estadounidenses. Las técnicas empleadas se exportan a Europa y a los finales de los setenta se utilizaron para estructurar una serie de financiaciones de proyectos de gran envergadura, casi todas ellas destinadas a plataformas petroleras del Mar del Norte ${ }^{3}$.

Esta técnica de financiación de proyectos se ha utilizado también en las líneas férreas en Argentina en 1860, la India 1880, el Canal de Suez en 1850, etc. Así también este mecanismo permitido se materialice múltiples proyectos de carácter privado como centros de entretenimiento, centros comerciales, desarrollos inmobiliarios, etc.

\section{Aclaraciones necesarias}

Hay que esclarecer cualquier confusión que pueda existir entre: (1) El proyecto a financiar $\mathrm{y}$, (2) El project finance (contrato project finance).

El proyecto a financiar, es una estructura económica compleja y de inversión, la cual

3 ICEX. Op. Cit. p. 15 y 16 atraviesa por diferentes etapas, desde el momento en que los promotores idean el proyecto, la creación de la sociedad vehículo del proyecto (SPV), la obtención de la financiación por parte de las entidades financieras (bancos), la construcción del proyecto, la explotación del mismo y por último el pago del principal y los intereses.

Mientras que el project finance es el mecanismo de financiación del proyecto, el cual se materializa a través de un contrato, el contrato project finance, el cual se celebra entre la SPV y la entidad financiera.

\section{Naturaleza jurídica del Project finance}

El Project Finance no está regulado en el ordenamiento jurídico peruano, sin embargo, la naturaleza jurídica de este contrato se fundamenta en el Principio de la Autonomía Privada de la Voluntad. Conjuntamente este contrato se encuadra como un "Contrato bancario, atípico y de financiación”.

\section{Intervienen en el Project finance}

Esta modalidad de financiación de proyectos requiere la necesaria participación de los siguientes agentes: Promotores del proyecto (socios), sociedad vehículo del proyecto (SPV), entidades financieras (bancos), contratista o constructor, el operador del proyecto, administración pública; compañías de aseguradoras, asesores (financieros, jurídicos, ingeniería, medioambientales), proveedores, trabajadores, cliente final o usuario del proyecto

\section{Promotores del proyecto - socios}

Personas física o jurídica que con la finalidad de desarrollar un mismo proyecto y debido al elevado costo del mismo, se asocian entre 
varios promotores constituyendo la sociedad vehículo del proyecto (SPV) aportando, generalmente, entre el diez y el treinta por cien (10 - 30\%) del capital total requerido, proporcionar el suelo, la tecnología, la gestión de las operaciones, construcción, influencia financiera, contactos locales, transporte, suministros, etc., los objetivos principales del promotor son atraer fuentes de financiamiento, generar fondos suficientes para el proyecto, colocación y distribución de los riesgos entre las distintas entidades financieras prestamistas, aislar los riesgos del proyecto, etc.

Los promotores o socios habitualmente son firmas conocedoras y operadoras del sector y negocio del proyecto, aportan parte del capital y el know-how, tienen experiencia previa en proyectos similares, estrategia y de gestión.

\section{La sociedad vehículo del proyecto - SPV}

Los promotores del proyecto se agrupan a través de una empresa nueva e independiente tanto legal como económica, denominada Sociedad Vehículo del Proyecto (SPV), la que se constituye específicamente para ejecutar, administrar y explotar el proyecto.

La SPV se encarga de buscar la financiación necesaria, lo hará entre sus socios y en el mercado financiero, además, la SPV será quien celebré los contratos con la entidad financiera, el contratista, proveedores, aseguradoras, clientes, entre otros. Además, la naturaleza del project finance implica que la SPV opere con un elevado nivel de endeudamiento sobre sus recursos propios, por eso su principal función será gestionar y generar eficazmente los flujos de caja que la propia SPV requiere para cumplir con las obligaciones asumidas con las entidades financieras y, en su momento realizar el reparto de dividendos.

\section{Entidad financiera - Bancos}

Las entidades financieras son quienes proporcionan el dinero para la financiación ${ }^{4}$. Son intermediarios del mercado financiero y podrán variar conforme a la naturaleza del proyecto. Las formas más conocidas son las siguientes: Bancos nacionales o extranjeros, caja de ahorros, empresas financieras, que son otro tipo de intermediarios financieros, que sin ser bancos, ofrecen préstamos o facilidades de financiamiento en dinero. Es a través de un banco principal que establece los vínculos con las demás entidades financieras (bancos subordinados) con la finalidad de alcanzar los altos niveles de financiamiento, esta estructura financiera es denominada crédito sindicado. En algunas latitudes existen organismos o instituciones nacionales e internacionales que brindan apoyo económico a los inversores.

Los bancos celebran el acuerdo de financiación con la SPV; sin embargo, no sólo se limitan a ello, sino que además, son quienes diseñen la estructura de la operación de financiación, es decir, preparan el proyecto base modelo de financiero - ponen especial énfasis en los mecanismos a utilizar para cubrir los riesgos.

Asimismo, el banco puede exigir a la SPV que firme otros contratos (como los de seguros) o requerirle que, estos, tengan determinadas características, por ejemplo, en los casos que el promotor llega con un borrador de contrato de construcción, o con uno ya firmado, el banco puede exigir que se modifique para hacerlo bajo la modalidad por ejemplo de contrato "llave en mano".

En cuanto a las condiciones que aplicaran serán más onerosos que un crédito o présta-

4 Bieger Morales, Pablo y Alonso Orcada, María L. "Financiación, Cuadernos de Derecho para Ingenieros", IBERDROLA, 2010, Capitulo I, p. 6 
mo ordinario, teniendo en cuanta que el reembolso de la financiación y las tasas fijadas están sujetas y condicionadas al éxito del propio proyecto.

\section{El contratista o constructor}

Es la empresa encargada de la construcción o edificación del proyecto, responsable de toda la obra y, la obligada a realizar todas aquellas operaciones necesarias para la puesta en marcha de las instalaciones. El contratista se vincula a la SPV mediante el contrato de construcción, generalmente contrato "llave en mano".

\section{El operador}

El operador es la persona que está a cargo de la operación y, en muchos casos, también del mantenimiento de las instalaciones de la SPV. El Operador puede ser la propia sociedad vehículo del proyecto, normalmente uno de los promotores es elegido debido a la experiencia en la industria y su capacidad de llevar a cabo esa tarea o también puede ser un tercero especializado. De cualquier forma, sus obligaciones son delimitadas en el contrato de operación y mantenimiento. Este contrato permite aislar los riesgos propios de la SPV (respecto a la titularidad de sus activos), de aquellos riesgos derivados del funcionamiento del proyecto.

Lo que se busca mediante el operador es, asignar parte del riesgo de operación y mantenimiento a la empresa que realice este trabajo, aislando a la SPV y a los financistas de este riesgo.

En virtud del contrato de operación y mantenimiento, el operador, además, en algunos casos, pagara una cantidad fija o mínima a la SPV si el proyecto no produce lo que debe- ría, ya que de esta manera se asegura una productividad mínima, por ejemplo a través de una garantía de productividad, independientemente del rendimiento real del proyecto, con lo que esta asegura unos flujos estables. Además, es común utilizar modelos de incentivos/penalidades, ofreciendo mejores perspectivas de que el proyecto sea operado eficientemente ${ }^{5}$.

\section{Beneficios}

Asegurar que las instalaciones se operen y mantengan dentro de los presupuestos acordados en el modelo económico y maximizar la capacidad de generación de flujos de caja del proyecto.

Las tareas de operación y mantenimiento requieren, en muchas ocasiones, realizarse en horarios amplios (turnos de 24 horas para la operación, noches, fines de semana y periodos vacacionales para determinadas tareas de mantenimiento) por ello se requerirá un aumento y disminución de plantilla, dependiendo de los trabajos a realizar. Los contratos de O\&M trasladan estos problemas fuera de la SPV, viendo así reducida su conflictividad laboral.

\section{La administración pública}

La administración pública juega un rol trascendente para la implementación de proyectos en los que se utiliza project finance como sistema de financiación. Los vínculos se darán en todo momento, desde que se constituye la SPV pues necesitara para su funcionamiento inscribirse en el registro de personas jurídicas; el proyecto también requerirá diversas autorizaciones, por ejemplo las exigidas en el régimen de la competencia; así como licencias

5 Menéndez, Aurelio; Rojo, Ángel, “Lecciones de De-
recho Mercantil” Vol. I y II, CIVITAS-THOMSON
REUTERS, Pamplona 2011, p. 221 
emitidas por las municipalidades, gobiernos regionales o centrales; la administración también está presente en lo referente al régimen tributario y el correspondiente pago de impuestos; incluso, en algunos casos el Estado es quien determina el control de cambio ${ }^{6}$; asimismo, los gobiernos tienen una vital influencia respecto al riesgo político o riesgo país, lo que implica un ámbito de evaluación que realizan los promotores respecto a la estabilidad del proyecto en el tiempo.

En los proyectos de Asociación Público-Privada - APP el vínculo se expresa a través del contrato de concesión, por el cual la SPV se obliga a la construcción de un determinado proyecto a cambio de su posterior explotación por un periodo de tiempo, como en el caso de las autopistas, puentes, túneles, canales, tendidos ferroviarios, etc.

Cabe agregar, la administración pública cuando participa en proyectos del tipo APP, por lo general lo hará aportando fincas de su titularidad, además, no es frecuente, pero también podrá aportar capital, con cargo a su presupuesto o también mediante la emisión de bonos en el mercado secundario, los que se encontraran garantizados por la misma administración.

\section{Las empresas aseguradoras}

Cobran también especial relevancia debido a que cubren aquellos riesgos difíciles de controlar por la SVP, como desastres naturales, estallido de guerra, etc.

6 Control de Cambio es un instrumento de política cambiaria que consiste en regular oficialmente la compra y venta de divisas en un país. De esta manera, el Gobierno interviene directamente en el mercado de moneda extranjera, controlando las entradas o salidas de capital.

\section{Los asesores externos del proyecto}

Toda operación de financiación de proyectos requiere un asesoramiento externo que permite acortar los riesgos del proyecto y estructurar la operación a financiar. Evalúan todas las características del proyecto: riesgos, estructura financiera, viabilidad económica y jurídica rentabilidad y cualquier otro aspecto que permita proteger el proyecto. En concreto, son necesarios:

\section{a. Asesores financieros:}

Encargados de valorar los riesgos económicos y financieros del proyecto.

\section{b. Asesores jurídicos:}

Toda operación de financiación de proyectos requiere la opinión legal, tributario, que evalúa el marco legislativo vigente y la posible afectación del proyecto. El asesor legal es necesario desde la fase inicial del proyecto, con el objeto de asegurar que se ha escogido el modelo optimo de gestión de la SPV. Además, asesora en los contratos entre los distintos agentes que intervienen en el proyecto.

\section{c. Asesores de ingeniería}

Su actividad consiste en realizar el estudio técnico independiente que permita valorar la viabilidad técnica del proyecto, su nivel de rendimiento esperado y los riesgos previsibles de la explotación.

\section{d. Asesores medioambientales}

Evalúan el impacto ambiental del proyecto, plantea alternativas a problemas, determina y evalúa riesgos con el entorno donde se ejecuta el proyecto. 
Otros asesores, en determinados proyectos son precisos informes de expertos independientes, como por ejemplo: Estudios de mercado, que evalúen la evolución esperada del sector y del mercado en el que se desarrolle su actividad la SPV. Agencias de rating, que califiquen posibles emisiones de deuda garantizada por parte de la SPV. Evaluación de seguros de todo tipo. La participación de estos asesores implica que el coste de estudio de este tipo de proyectos sea más elevado que el de otros proyectos financiados por otros sistemas más tradicionales.

e. El cliente final o usuario

Son aquellas personas que se comprometen a adquirir y pagar el producto o servicio generado una vez que se encuentre el proyecto en la etapa de funcionamiento, obligándose mediante un contrato de compra venta. Este contrato será el eje central para la obtención de flujos de caja o ingresos, asegurando de esta manera la sostenibilidad del proyecto.

Cuando se trata de Asociación Público-Privada, por ejemplo, la construcción de una central eléctrica, podrá ser la Administración quien se obligue a ser el principal adquirente de la energía producida en dicho proyecto.

\section{Diez características del Project finance}

1. El proyecto tiene que ser claramente identificable como actividad independiente de cualquier otra actividad desarrollada por alguno de los participantes del proyecto.

2. Los integrantes del proyecto deben tener solvencia tanto económica como técnica y una capacidad de gestión probada.
3. Los flujos de caja que vaya a generar el proyecto deberán ser predecibles y estables. Éste es el caso de los proyectos relacionados con la electricidad, el petróleo, el gas, las autopistas o el agua, por ejemplo, la rentabilidad de una infraestructura destinada al ocio; el éxito de la propuesta dependerá de la moda y de diversos factores que introducirían un mayor riesgo e inestabilidad.

4. El entorno legal, económico y social en el que se desarrolla el proyecto debe de ser adecuado y estable.

5. El project finance es de gran utilidad para los promotores de proyectos, toda vez que, su inversión se encuentra aislada en una persona jurídica cuyo objeto social será la realización y explotación del propio proyecto.

6. El banco acreedor, al otorgar la financiación lo hace sin recurrir, por lo general, a garantías adicionales a las que proporciona el propio proyecto, ya que la SPV asume como deudor una responsabilidad universal a través de todos sus bienes (los activos y derechos del proyecto), esto se debe a que el banco espera que el proyecto sea capaz de generar futuros flujos de efectivo, para de esta manera asegurar la devolución de la deuda y los intereses correspondientes. Por ello se dice que el propio proyecto garantizara la financiación, sin que el promotor ponga en riesgo más que lo que aporta.

7. Los bancos proveen financiación a un proyecto singular y asumen una gran parte del éxito o fracaso de ese proyecto. Lo que se espera es que el proyecto una vez se encuentre operativo en la etapa de explotación, sea capaz de devolver la deuda y los in- 
tereses correspondientes a la entidad financiera.

8. El project finance se utiliza en la construcción de grandes proyectos, en el sector privado, como en el sector público, en este último caso, debido a que los sistemas tradicionales de financiación han resultado insuficiente el sector privado interviene y participa en la construcción de grandes proyectos de dominio público bajo la forma PPPs, Public-Private Partnership (Colaboración Público Privada).

9. El Project Finance implica la realización de contratos entre los participantes, en los que se definan las funciones, riesgos y responsabilidades y se regulen las relaciones que tengan.

10. Este tipo de financiación, si bien es cierto tiene como premisa que el promotor no pondrá en riesgo más bienes que los que aporta el proyecto, y que por ende no asume responsabilidad personal por la deuda; sin embargo, en la práctica, en algunos casos si es posible que se pidan garantías a los socios promotores en periodo como el de construcción o, en determinados supuestos en los que claramente se encuentren bajo su control. También es posible que se establezcan mecanismos de apoyo por parte de los socios, aunque jurídicamente no sean garantía, ya que se consideran aportaciones de fondos adicionales contingentes para cubrir sobrecostes, penalidades, etc.

\section{Fases especificas en el Project finance}

Un proyecto que se financia mediante la modalidad project finance se desarrolla a través de tres fases claramente diferenciadas:

\section{Fase previa:}

Durante esta etapa, se estudia la posibilidad de realizar el proyecto empleando diversas técnicas. Una vez decidido se planifica en detalle el mismo. La planificación del proyecto incluye, entre otros aspectos, la búsqueda de las mejores soluciones técnicas, la búsqueda de los inversores y métodos de financiación, así como la anticipación de los posibles contratiempos que puedan ocurrir.

En el caso que, tras el estudio de viabilidad se opte por no realizar el proyecto, el desembolso incurrido durante esta etapa se habría hecho a fondo perdido.

\section{Fase de construcción:}

Durante la fase de construcción se continúa incurriendo en flujos de caja negativos, existen mayores riesgos e intervienen un mayor número de agentes. Es la etapa que tiene una estructura más compleja. La construcción de la instalación, por lo general se adjudica a un único contratista mediante lo modalidad de contrato "llave en mano". Este contratista principal realizará parte de los trabajos, subcontratando a su vez otra gran parte de los mismos a terceras subcontratas. La estructura se repite creando una red de subcontratos y englobando la totalidad de los trabajos. La financiación de la estructura es igualmente complicada pudiendo existir de manera análoga a la estructura de subcontrataciones una red de sindicaciones entre los distintos agentes financieros.

\section{Fase de explotación:}

En esta fase se obtienen los flujos de caja positivos que justifican los costes incurridos en las etapas anteriores e incluyen el beneficio de los inversores. Durante este periodo tam- 
bién existen costes de operación y mantenimiento propios del funcionamiento del negocio. Al final del mismo se incurre en los costes de desmantelamiento de las instalaciones, que significarán unos últimos flujos de caja negativos que hay que considerar en todo momento ${ }^{7}$.

\section{Consideraciones finales}

1) Sí el proyecto, una vez que se encuentre en la etapa de operación o funcionamiento logra alcanzar significativos flujos de cash flow generándose ciertos excedentes económicos, si llegase a ocurrir ello, previamente se debe haber establecido una clausula contractual para la creación de un fondo de reserva, esto significa que sí se generan mayores ingresos, estos, tendrán que acumularse en el citado fondo, el cual impedirá que los promotores prioricen el reparto de dividendos ante los inusuales y elevados ingresos que el proyecto genere en un momento determinado; en la práctica esto significa poner en riesgo la cancelación del crédito. Al crearse este fondo de reserva la entidad financiera incrementa su respaldo ante posibles futuros riesgos de impago.

2) Durante la construcción del proyecto se suscitan una multitud de riesgos, siendo relevantes aquellos que tienen un impacto en el plazo o en el costo de las instalaciones. El impacto en plazo implica un retraso en el comienzo de la generación de los flujos de caja positivos, y por tanto un retraso en la devolución del préstamo, es decir un aumento de los costos financieros. Los costos asociados con los impactos deberán ser asumidos por alguno de los integrantes del proyecto en función del factor que haya provocado el riesgo. Si el retraso o el extra-costo han sido motivados por errores en el diseño o en la planificación del proyecto, el costo lo debe asumir el constructor del proyecto: esto se articula mediante el contrato "llave en mano", donde el precio es fijo y asociadas a retrasos en la finalización del proyecto aparecen penalizaciones. Sí el factor es incontrolable o accidental es común la contratación de seguros. También si la situación límite, si el riesgo significase la no realización del proyecto, el impacto principal recaería en las entidades financieras, procediendo estas a liquidar la sociedad vehículo del proyecto, vendiendo sus activos e iniciando acciones legales sobre los responsables de la situación en el caso de que esto fuera posible.

3) De esta manera concluye la presente investigación teniendo en cuenta que el project finance debe estructurarse observando correctos mecanismos de protección contra el riesgo para el efectivo cumpliendo de la financiación otorgada por la entidades financieras, esto directamente permitirá incentivar a las entidades financieras nacionales y extranjeras su participación en esta modalidad de financiación, lo que conllevaría al incremento en la construcción diversos proyectos de inversión e infraestructura resultando esto, un beneficio para los empresarios, entidades financieras y la sociedad en su conjunto. 
